\title{
Biological Indexes Based Reflectional Asymmetry for Classifying Cutaneous Lesions
}

\author{
Zhao Liu ${ }^{1}$, Lyndon Smith ${ }^{1}$, Jiuai Sun ${ }^{1}$, Melvyn Smith ${ }^{1}$, and Robert Warr ${ }^{2}$ \\ ${ }^{1}$ Machine Vision Lab, University of the West of England, Bristol, UK \\ ${ }^{2}$ Plastic Surgery, Frenchay Hospital, NHS, Bristol, UK
}

\begin{abstract}
This paper proposes a novel reflectional asymmetry descriptor to quantize the asymmetry of the cutaneous lesions for the discrimination of malignant melanoma from benign nevi. A pigmentation elevation model of the biological indexes is first constructed, and then the asymmetry descriptor is computed by minimizing the histogram difference of the global point signatures of the pigmentation model. Melanin and Erythema Indexes are used instead of the original intensities in colour space to characterize the pigmentation distribution of the cutaneous lesions. 311 dermoscopy images are used to validate the algorithm performance, where $88.50 \%$ sensitivity and $81.92 \%$ specificity have been achieved when employing an SVM classifier.
\end{abstract}

\section{Introduction}

An abnormal reproduction of biological cells within body organs and tissues usually forms an asymmetric shape or appearance, with a potential to become metastatic and aggressive spreading thorough the body. Malignant melanoma, which accounts for $75 \%$ mortality caused by skin cancers [1], is one of these examples. Asymmetry therefore has been demonstrated as one of the most important features to quantify the shape and structure of the lesion. Stoecker et al. [2] determined the reflectional asymmetry of the lesion by area differences across the principal axes computed from the moment of inertia. Similarly, Seidenari et al. [3] evaluated the lesion asymmetry by calculating the area differences with respect to 128 axes through the lesion centre. Stanganelli et al. [4] applied the size function to separately quantize the asymmetry of the lesion in terms of boundary, shape and colour to achieve a better classification results.

Most of the above approaches have shown the discrimination power of asymmetry in melanoma identification. However, they share some similar shortcomings. Firstly, most of the existing asymmetry descriptors only evaluate the extrinsic shape, but ignore the asymmetry of the inhomogeneous pigmentation inside the lesion. Secondly, since asymmetry measure and symmetry axis are normally defined separately, the final results might not be optimized. Thirdly, clinically acquired lesion images may contain complex distortions due to the factors like sensor positions and lighting conditions. Normal extrinsic asymmetry descriptors greatly vary with them.

This paper proposed a new intrinsic reflectional asymmetry descriptor to simultaneously quantize the shape and the pigmentation distributions of the cutaneous 
lesions with robustness to various deformations. We compute the melanin index and erythema index from an RGB image, and then map these biology values to the Z-axis to build a pigmentation elevation model. The reflectional asymmetry of the lesion is defined by minimizing the histogram difference of the global point signatures (GPSs) of the pigmentation model. Compared with those shape dependent asymmetry detectors, the proposed asymmetry descriptor is more efficient in describing the abnormality of the lesion as it simultaneously integrates the shape and pigmentation information. Moreover, melanin and erythema indexes reveal the pathological tissue conditions, which proved more useful in characterizing the irregularity of the pigmentation of the cutaneous lesions. We have verified that the proposed GPSsbased asymmetry descriptor is invariant to $2 \mathrm{D}$ rigid transformations. It is also robust to the non-rigid deformations, which is ideal for characterizing the intrinsic asymmetry of the skin lesions.

\section{Biology Pigmentation of the Skin Lesions}

Melanin and haemoglobin are two primary chromophore components determining the colour of human skin. Excessive ultraviolet radiation from the sun may cause irregular melanin growth in horizontal and vertical directions. When the aggressive melanocytes reach the vascular system, they become malignant and are easily broadcast to the whole body. Therefore the irregularity of both melanin and haemoglobin are internal factors for skin cancer diagnosis, though they are normally presented as colour values acquired by optical cameras.

Melanin Index (MI) and Erythema Index (EI), reflecting the pigmentation and vascular blood status of human skin, can be approximated from Red and Green channels of an RGB image. Regarding the skin reflectance is in Green $(\sim 560 \mathrm{~nm})$ (high hemoglobin absorption) and Red ( 650nm) (low hemoglobin absorption) spectral ranges, the approximations of MI and EI can be defined [5] as,

$$
\begin{gathered}
\text { MI }=100 * \log _{10}(1 / R) \\
\text { EI }=100 *\left(\log _{10}(1 / \mathrm{G})-1.44 * \log _{10}(1 / R)\right)
\end{gathered}
$$

where $\mathrm{R}$ and $\mathrm{G}$ are intensities in red and green channels respectively. Fig.1 shows the $\mathrm{MI}$ and EI mappings of a malignant melanoma (MM) and a benign nevus (BN).

(i) $\mathrm{MM}$

(ii) $\mathrm{BN}$

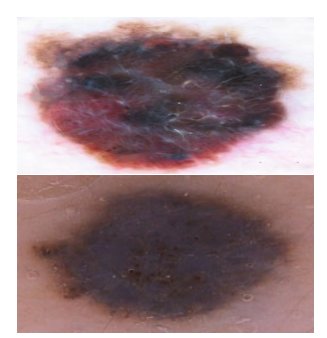

(a) Original images

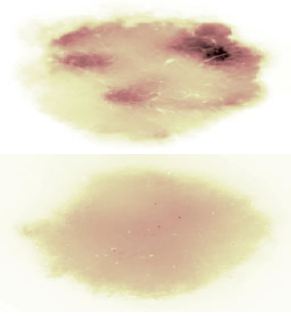

(b) $\mathrm{MI}$

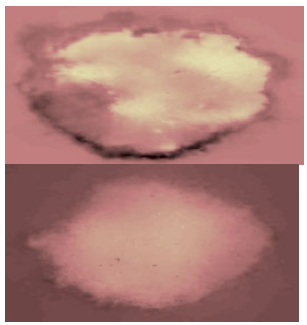

(c) $\mathrm{EI}$

Fig. 1. Melanin and Erythema mappings of (a) MM, (b) BN 


\section{Asymmetry Descriptor}

\subsection{Pigmentation Elevation Model}

A few pre-processing steps are applied to remove the hairs on the skin [6], flatten the homogeneous regions, and isolate the lesions from the surrounding normal skin [7]. Then MI and EI are calculated to build the pigmentation elevation model by mapping $\mathrm{MI} / \mathrm{EI}$ to Z-axis, and the shape information of the isolated lesion as $\mathrm{X}$ and $\mathrm{Y}$ axes.

In order to enable the shape and pigmentation giving the comparable contributions, the pigmentation model is scaled down in a bound box. Suppose $\mathrm{X}_{\text {lesion }}$ has larger range than $\mathrm{Y}_{\text {lesion }}$, then they are first normalized as $\mathrm{X}_{\text {lesion }}^{\prime}=\mathrm{X}_{\text {lesion }} /\left(\max \left(\mathrm{X}_{\text {lesion }}\right)-\right.$ $\left.\min \left(\mathrm{X}_{\text {lesion }}\right)\right)$ and $\mathrm{Y}_{\text {lesion }}^{\prime}=\left(\mathrm{r} * \mathrm{Y}_{\text {lesion }}\right) /\left(\max \left(\mathrm{Y}_{\text {lesion }}\right)-\min \left(\mathrm{Y}_{\text {lesion }}\right)\right)$. As a result, the range of $\mathrm{X}_{\text {lesion }}^{\prime}$ is 1 , and that of $\mathrm{Y}_{\text {lesion }}^{\prime}$ is $r$, where $r=\mathrm{Y}_{\text {lesion }} / \mathrm{X}_{\text {lesion }}$. Next normalize $\mathrm{Z}$-axis to the range of [0 1]. Because the maximum and the minimum values in MI and EI vary among images, conventional normalization yields non-uniform results. So we calculate the range of $\mathrm{MI}$ and EI indexes according to (1) and (2), where the range of MI is [-240.82, 0] and that of EI is [-240.82, 346.78]. Then the normalized EI and MI images can be calculated below to ensure the effectiveness of the pigmentation normalization is equivalent to every single image,

$$
\begin{aligned}
& M I_{\text {norm }}=\frac{M I-(-240.82)}{240.82}=0.0042 M I+1 \\
& E I_{\text {norm }}=\frac{E I-(-240.82)}{587.6}=0.0017 E I+0.4098
\end{aligned}
$$

Fig. 2 shows the pigmentation elevation models calculated from MI and EI in Fig.1. The outer boundary of the model stands for the shape asymmetry of the skin lesion, and the pigmentation asymmetry of the lesion can be reflected by the distribution of the pigmentation model along the Z-direction.

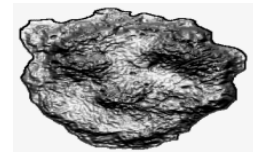

(a) model in MI

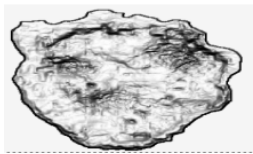

(b) model in EI

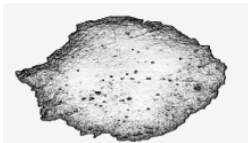

(c) Model in MI

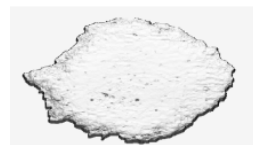

(d) model in EI

(i) $\mathrm{MM}$

(ii) $\mathrm{BN}$

Fig. 2. Pigmentation elevation model from lesion images in Fig.1. (i) MM, (ii) BN

\subsection{Reflectional Asymmetry in Histograms}

The proposed GPSs-based asymmetry descriptor is derived from the Laplace-Beltrami operator, which is defined as the divergence of the gradient on a surface function in Riemannian manifold M. The Laplace-Beltrami operator L can be approximated by the graph Laplacian matrix using the heat kernel [8], where the eigenvectors of the Laplacian matrix embed the points on $\mathbf{M}$ into lower dimensional representations. Since the Laplace-Beltrami operator is symmetric negative and semidefinite, it has an eigen-decomposition $L \emptyset_{\mathrm{i}}=\lambda_{\mathrm{i}} \emptyset_{\mathrm{i}}\left(0=\lambda_{0}<\lambda_{1}<\cdots<\lambda_{i}\right)$, where $\emptyset_{\mathrm{i}}$ are eigenfunctions and $\lambda_{\mathrm{i}}$ are the corresponding eigenvalues. The GPSs can then be calculated [9] as: 


$$
\text { gps }=\left(\frac{\emptyset_{1}}{\sqrt{\lambda_{1}}}, \frac{\emptyset_{2}}{\sqrt{\lambda_{2}}}, \frac{\emptyset_{3}}{\sqrt{\lambda_{3}}}, \ldots, \frac{\emptyset_{i}}{\sqrt{\lambda_{i}}}, \ldots\right)
$$

GPSs are used because they can integrate shape and colour asymmetry detections on the pigmentation model simultaneously. Moreover, it has been proved that GPSs reflect the intrinsic metric property of the object and are robust to the metric deformations [9] which might be introduced by the camera positions or various lighting conditions during the data acquisition.

In our work, $\mathrm{M}$ is the pigmentation model in 3-dimensional space. The eigenvectors corresponding to the first few eigenvalues determine the optimal embeddings. Since the first eigenvector with $\lambda_{0}=0$ generates a constant function and the eigenvectors associated with repeated eigenvalues are not stable for small nonisometric perturbations [9], we restrict our searching for reflectional asymmetry detection in the first 6 eigenfunctions with non-zero and non-repeated eigenvlaues.

GPSs uniquely determine the metric of the manifold. So $M$ is intrinsically symmetric if there is an associated self-mapping $\mathrm{T}: \mathrm{M} \rightarrow \mathrm{M}$, making both $\emptyset_{\mathrm{i}}$ and $\emptyset_{\mathrm{i}} \circ \mathrm{T}$ the eigenvectors of $L$. Suppose $g p s_{i}$ is the $\mathrm{i}^{\text {th }}(1 \leq \mathrm{i} \leq 6)$ component in the GPSs, and $\mathrm{T}=\left\{\mathrm{t}_{1}, \mathrm{t}_{2}, \ldots, \mathrm{t}_{6}\right\}$ is the self-mapping. For a complete symmetry object, the GPSs with non-repeated eigenvalues only holds two possibilities along the reflectional symmetry axis as $g p s_{i} \circ T_{i}=g p s_{i}$ and $g p s_{i} \circ T_{i}=-g p s_{i}$. Thus $\mathrm{T}$ can be determined by a sign sequence with either positive $(+1)$ or negative $(-1)$. Skin lesions are imperfect or nonsymmetric objects, so the complete symmetry measure $|g p s \circ T|=|g p s|$ could not be fulfilled. Thus we generate a region-based reflectional asymmetry descriptor in histogram to quantify the asymmetry of the skin lesions in the GPS spaces.

We first defined the gravity centre of a lesion, and then the entire lesion is segmented into 180 segments around the polar coordinate across the centre. For each segment, we built the histogram of GPS with 100 bins, thus the descriptor of each segment $l$ in signature $i$ can be represented as (6).

$$
\operatorname{Des}_{i, l}(T)=\frac{1}{\mathrm{~N}_{\mathrm{l}}} \sum_{\mathrm{n}=1}^{100} f\left(g p s_{i, l}^{n}(T)\right) * v\left(g s_{i, l}^{n}(T)\right) \quad l=1,2, \ldots, 180
$$

where $f$ represents the frequency counts in each bin, $\mathrm{v}$ is the bin location of $g p s_{i, l}(T)$ in histogram and $\mathrm{N}_{\mathrm{l}}$ is the number of pixels in each segment. For every $\operatorname{gps} s_{i}(T)$, the $\operatorname{Des}_{i}(T)$ value can be plotted from 0 to $\pi$. As the principal axis must exist in these 180 segments, we assume one segment as the principal axis at each time, and translate part of the $\operatorname{Des}_{i}(T)$ to ensure 90 elements on both sides along the axis. Considering there are six GPSs, the asymmetric degree of a lesion can be quantified by minimizing the Euclidean distance between the left-right sides of the histogram,

$$
\operatorname{Asy}(T)=\min \left(\sum_{i=1}^{6} \sum_{l=1}^{90}\left\|\operatorname{Des}_{i, l}^{L}(T)-\operatorname{Des}_{i, l}^{R}(T)\right\|_{2}^{2}\right)
$$

where $\operatorname{Des}_{i, l}^{L}$ represents the left side and $\operatorname{Des}_{i, l}^{R}$ stands for the right part of the histogram. Because the asymmetry descriptor is a function of the sign sequence $T$, the minimum asymmetry measure also indicates the potential optimal reflectional axis. Moreover, since the GPSs integrate the shape and pigmentation asymmetry detections simultaneously, it avoids yielding two different symmetry planes when the asymmetric appearance of shape and colour are analyzed separately.

Fig.3(i) shows the first 6 GPSs with the optimal sign sequence $\mathrm{T}$ from the MI images of the lesions in Fig.1. The BN shows approximate symmetric appearance in a 
given direction for all $\operatorname{gps}(T)$, whereas it is difficult to find an appropriate symmetry plane fit for the six $g p s(T)$ of the MM. The first two $g p s(T)$ indicate shape asymmetry, while higher orders of $g p s(T)$ reflect the pigmentation distribution inside the lesion. Fig.3(ii) plots the optimal translated histograms with the minimum asymmetry measure. The BN shows symmetry on left-right side of the histograms, while the optimal translated histograms of the MM greatly fluctuate. It results in a large asymmetry measure which can be used for the classification purpose.

(i)

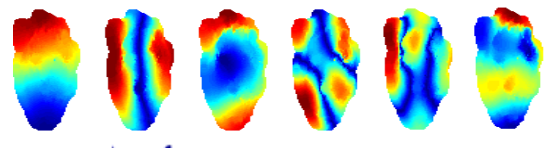

(ii)

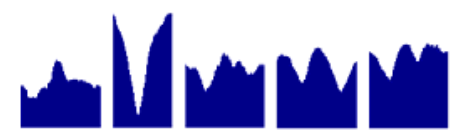

(a) $\mathrm{MM}$
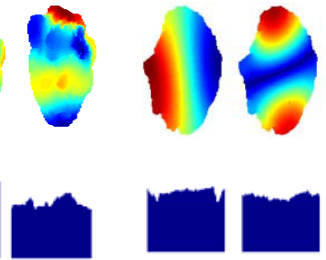
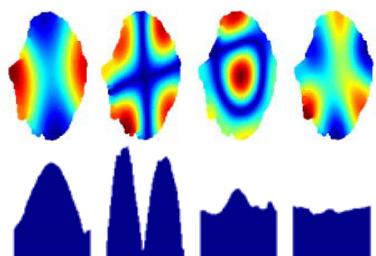

(b) $\mathrm{BN}$

Fig. 3. Asymmetry descriptors of the MM and the BN in Fig.1. (i) The first 6 GPSs with the optimal sign sequence T. (ii) Translated histograms given the minimum asymmetry measures.

\subsection{Influence of Rigid Transformations and Non-rigid Deformations}

One of the important characteristics of the asymmetry descriptors for skin lesion is whether it is robust to the rigid transformations (translation, rotation, scale) and nonrigid deformations. Next we will evaluate their influence to our asymmetry descriptor.

Invariant to Rigid Transformations and Isometric Deformations. GPSs are calculated from graph the Laplace matrix by connecting the neighbour points on object surface in Riemannian space. It has been proved that GPSs are invariant to translation, rotation and isometric deformations [8], because these manipulations do not change the point distance along the surface. Since histogram neglects the spatial information and does not introduce extra isometric deformations, the proposed asymmetry descriptor is translation, rotation and isometric deformation invariant.

For scaling, suppose a ratio $\gamma$ scaled the manifold $\mathrm{M}^{2} \mathrm{M}^{\prime}=\gamma \mathrm{M}$. Eigenvalues and eigenvectors of $\mathrm{M}^{\prime}$ can then be calculated as $\lambda_{\mathrm{i}}^{\prime}=\lambda_{\mathrm{i}} / \gamma^{2}$ and $\emptyset_{\mathrm{i}}^{\prime}=\emptyset_{\mathrm{i}} / \gamma$. GPSs of the scaled $\mathrm{M}^{\prime}$ proved invariant since $\mathrm{GPS}_{\mathrm{i}}{ }^{\prime}=\emptyset_{\mathrm{i}}^{\prime} / \sqrt{\lambda_{\mathrm{i}}^{\prime}}=\left(\emptyset_{\mathrm{i}} / \gamma\right) / \sqrt{\lambda_{\mathrm{i}} / \gamma^{2}}=\emptyset_{\mathrm{i}} / \lambda_{\mathrm{i}}=\mathrm{GPS}_{\mathrm{i}}$.

Considering parameter $\mathrm{m}, \mathrm{l}$ and sign sequence $\mathrm{T}$ are scale irrelevant, only descriptor $D e s_{i, l}(T)$ in (6) needs checking. Since GPSs are scale invariant, bin location v, representing the quantified value in GPS histograms, stays unchangeable to $\gamma . f$ representing the frequency counts, is a function of pixel number. As such dividing the pixel number $\mathrm{N}_{1}$ in each segment as (7) can counteracts the influence of $\gamma$.

Robustness to Non-isometric Deformations. Since skin lesions are non-rigid objects, they associate with non-isometric deformations most of the time. Besides the 
2D shape distortions due to skin surface tension, non- isometric deformations in Zaxis also exist because of the different intensity caused by different dermoscopes.

Given a d-dimensional manifold $\mathrm{M}$ and its non-isometric deformation $\mathrm{M}^{\prime}$, the graph Laplace matrix can be represented as $L=S^{-1} C$ and $L^{\prime}=S^{\prime-1} C^{\prime}$, where $S$ represents the area surrounding by a pixel's neighbour points, and $\mathrm{C}$ is a cotangent function [9]. $\emptyset_{\mathrm{i}}^{\prime}$ and $\lambda_{\mathrm{i}}^{\prime}$ of the non-isometric perturbation object will remain stable if non-isometric perturbation $\Delta C=\left|C-C^{\prime}\right|$ and $\Delta S=\left|S-S^{\prime}\right|$ are small. Thus the proposed asymmetry descriptor is robust to small non-isometric deformations.

\section{Experimental Results}

The performance of the asymmetry descriptors for melanoma diagnosis is validated on 311 dermoscopy images [10][11] with resolution ranging from $448 \times 336$ to $1098 \times 826$ pixels, where there are $88 \mathrm{MMs}$ and $223 \mathrm{BNs}$. The asymmetry descriptor is a four dimensional feature vector including the minimum asymmetry measures obtained from MI and EI images respectively, as well as their asymmetry measures in the direction perpendicular to the optimal reflectional axis.

$$
\text { Asymmetry Features }=\left\{A s y_{\min }(M I), A s y_{\min +90^{\circ}}(M I), A s y_{\min }(E I), A s y_{\min +90^{\circ}}(E I)\right\}
$$

\subsection{Efficiency of Biology Information}

In order to demonstrate the efficiency of the biology information, we compute the asymmetry descriptor in each channel of the conventional RGB images, and compare the diagnosis results with those from the melanin and erythema indexes.

Fig.4 plots the distributions of the probability density functions (pdfs) of the proposed asymmetry descriptors obtained from MI/EI images, as well as those from the conventional RGB images. The asymmetry descriptors in MI/EI images give better separated distributions between MMs and BNs. The application of the biology descriptors increases the discrimination, because MI/EI reflects the pathological condition and the primary cause of the pigmentation inside the lesion. This makes MI and EI ideal descriptors for quantizing of the malignancy of the skin lesions.

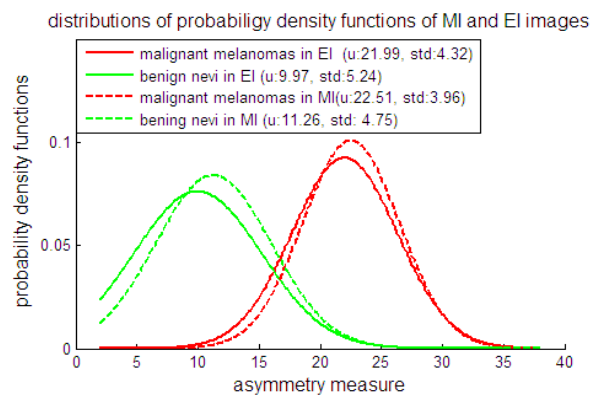

(a)

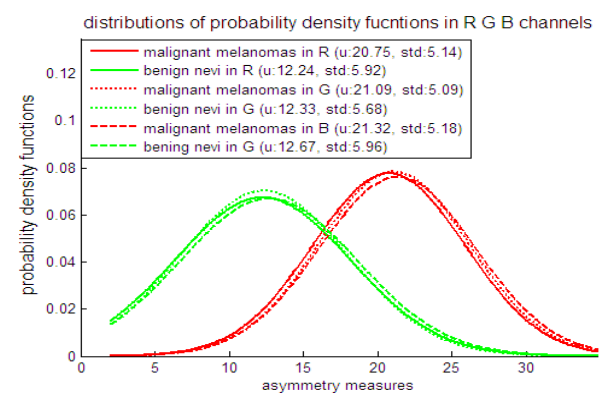

(b)

Fig. 4. Distributions of probability density function (pdfs) of the asymmetry descriptors. (a) pdfs in $\mathrm{MI}$ and EI images. (b) pdfs in Red, Green and Blue channels in conventional RGB images. 


\subsection{Robustness to Non-isometric Deformations}

To demonstrate the robustness of the proposed asymmetry descriptor to the nonisometric deformations, we calculate the asymmetry descriptors of the same lesion acquired by different dermoscopes. An example of Seborrheic Keratosis is shown in Fig.5. Although the overall shape of the lesion seems unchanged, non-uniform surface tension will always exist, not to mention the different lesion boundaries introduced by the automatic segmentation techniques. Thus $2 \mathrm{D}$ shape distortion between two images can be detected from the isolated lesions in Fig.5(ii). Moreover, compared with the original images, colour contrast in two MI images proves similar. Similar contrast with different intensity values only causes a translation along the Z-axis in the pigmentation model, but not greatly distorted the shape. So we assume there are small non-isometric deformations between the post-processed images.

Though both shape and colour distortions are presented, the asymmetry of the first 6 GPSs in Fig.5(ii) appear resembled. This similarity can be also observed from the corresponding histograms. Numerically, asymmetry degree of the lesion in (a) is 8.53, whereas that of the lesion in (b) is 9.27 , which gives $8.87 \%$ difference.

(i)
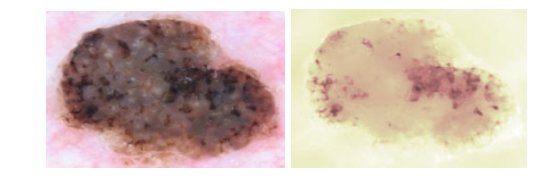

(ii)

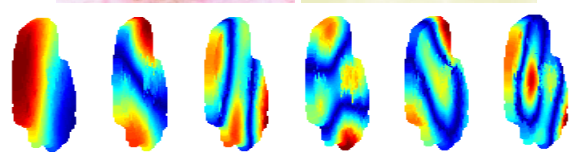

(iii)

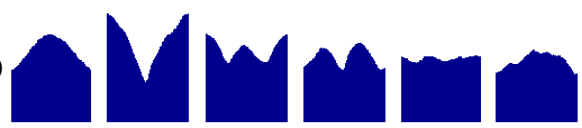

(a)

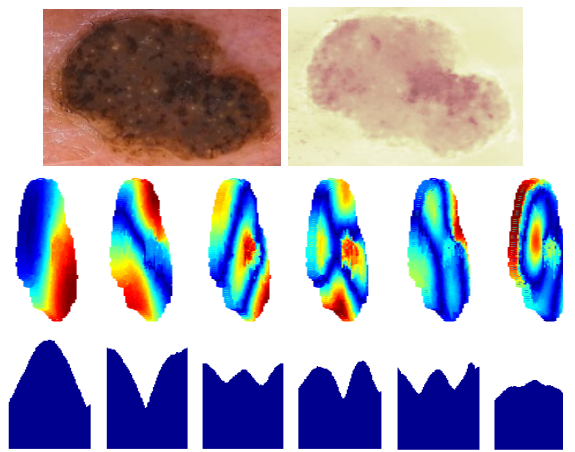

(b)

Fig. 5. Asymmetry measure of the same seborrheic keratosis by different dermoscopes. (a) polarized dermoscopy with resolution of $598 \times 492$ (b) immersion contact dermoscopy with the resolution of $540 \times 462$. (i) Original images and MI images. (ii) The first 6 GPSs with the optimal sign sequence T. (iii) Translated histograms given the minimum asymmetry measures.

\subsection{Performance of the Asymmetry Descriptors}

In order to demonstrate the efficiency of the proposed asymmetry descriptor, we compute the extrinsic shape and colour asymmetry without GPSs, and compare the classification results with that from the intrinsic descriptors with GPSs. The asymmetry measures without GPSs are defined similarly as the proposed detector. Specifically, a lesion is first segmented into 180 areas. Then each segment is represented by the area proportion of the segment to the whole lesion $\left(S D e S_{l}\right)$, or by the MI/EI histogram $\left(C D e s_{l}\right)$. Finally the asymmetry measure without GPSs can be quantified by minimizing the histogram difference in $[0 \pi]$ as $(9)$. 


$$
\operatorname{SDes}_{l}=\frac{N_{l}}{N}, \quad \operatorname{CDes}_{l}=\frac{1}{N_{l}} \sum_{n=1}^{100} f\left(\operatorname{colour}_{l}^{n}\right) * v\left(\operatorname{colour}_{l}^{n}\right)
$$

$$
S A s y=\min \left(\sum_{l=1}^{90}\left\|S \operatorname{Des}_{l}^{L}-\operatorname{SDes}_{l}^{R}\right\|_{2}^{2}\right), \quad C A s y=\min \left(\sum_{l=1}^{90}\left\|\operatorname{CDes}_{l}^{L}-\operatorname{CDes}_{l}^{R}\right\|_{2}^{2}\right)
$$

Three different classifiers were applied to validate the classification accuracy, including Support Vector Machine (SVM) with radial basis function of a scalar 3, Artificial Neural Networks (ANN) with 1 hidden layer and 5 neurons, and Bayesian classifier (BC) by minimizing the Bayesian risk of the 0/1-loss function. In the training-testing process, 155 dermoscopy images were randomly selected for training and the other half of data were used for testing. For each classification algorithm, we automatically execute the program 30 times and record the average sensitivity, specificity and accuracy as the final results to complement the bias introduced by the inconsistence of the random selection of the training data.

Table. 1 shows the classification results of the asymmetry descriptors with and without GPSs from each classifier. The accuracy from the GPSs-based asymmetry descriptor is approximate $5 \%$ higher than that of the combination of shape and colour asymmetry descriptors without GPSs. The best diagnosis for the test data are $88.50 \%$ sensitivity, $81.92 \%$ specificity and $83.26 \%$ accuracy employing the SVM classifier.

Table 1. Classification results of the asymmetry descriptors with and without GPSs

\begin{tabular}{|l|l|l|l|l|l|l|}
\hline & \multicolumn{3}{|l}{ Training (\%) } & with/without GPSs) & \multicolumn{3}{l|}{ Testing (\%) } & (with/without GPSs) \\
\cline { 2 - 7 } & Sensitivity & Specificity & Accuracy & Sensitivity & Specificity & Accuracy \\
\hline SVM & $94.53 / 93.02$ & $84.07 / 81.44$ & $87.03 / 84.72$ & $88.50 / 83.63$ & $81.92 / 75.81$ & $83.26 / 78.02$ \\
\hline ANN & $88.58 / 86.36$ & $80.49 / 77.82$ & $82.78 / 80.24$ & $86.27 / 82.92$ & $78.64 / 74.23$ & $79.96 / 76.69$ \\
\hline BC & $90.25 / 86.40$ & $81.10 / 76.19$ & $83.69 / 79.08$ & $83.35 / 79.56$ & $75.86 / 70.78$ & $77.98 / 73.26$ \\
\hline
\end{tabular}

\section{Conclusions}

This paper proposes a novel reflectional asymmetry descriptor by minimizing the histogram difference of the global point signatures on the pigmentation elevation model of the cutaneous lesions. Melanin and Erythema indexes have been proved more efficient than colour intensities in characterizing the pigmentation of the lesions. The proposed asymmetry descriptor is invariant to $2 \mathrm{D}$ rigid transformations and robust to non-isometric deformations. Competitive classification results of $88.50 \%$ sensitivity and $81.92 \%$ specificity have been achieved for melanoma diagnosis.

\section{References}

[1] Jerant, A., Johnson, J., Sheridan, C., Caffrey, T.: Early Detection and Treatment of Skin Cancer. American Family Physician 6, 357-368 (2000)

[2] Stoecker, W., Li, W., Moss, R.: Automatic Detection of Asymmetry in Skin Tumors. Computerized Medical Imaging and Graphics 16, 191-197 (1992)

[3] Seidenari, S., Pellacani, G., Giannetti, A.: Digital Videomicroscopy and Image Analysiswith Automatic Classification for Detection of Thin Melanomas. Melanoma Research 9, 163-171 (1999) 
[4] d'Amico, M., Ferri, M., Stanganelli, I.: Qualitative Asymmetry Measure for Melanoma Detection. In: IEEE Int. Symposium. Biomedical Imaging: Nano to Macro, pp. 11551158. IEEE Press, New York (2004)

[5] Bae, Y., Nelson, J., Jung, B.: Multimodal Facial Colour Imaging Modality for Objective Analysis of Skin Lesions. Journal of Biomed Optics 13(6), 064007 (2008)

[6] Lee, T., Ng, V., Gallagher, R., Coldman, A., McLean, D.: DullRazor: A software approach to hair removal from images. Computers in Biology and Medicine 27, 533543 (1997)

[7] Liu, Z., Sun, J., Smith, M., Smith, L., Warr, R.: An automatic mean-shift based segmentation for pigmented skin lesions. Medical Imaging Understanding and Analysis, 121-126 (2010)

[8] Balkan, M., Niyogi, P.: Laplace-beltrami Eigenfunctions for Deformation Invariant Shape Representation. Neural Computation 15(6), 1373-1396 (2002)

[9] Ovsjanikov, M., Sun, J., Guibas, L.: Global Intrinsic Symmetries of Shapes. In: Symposium on Geometry Processing, vol. 27, pp. 1341-1348. Eurographics Association, Aire-la-Ville (2008)

[10] Home page - Dr. Eric Ehrsam, http: / / dermoscopic.blogspot.com/

[11] Dermatology Information System, http: //www. dermis.net/ 